\title{
EMILIO GABBA E L'UNIVERSITÀ DI PAVIA
}

\author{
FABIO RUGGE (*)
}

SunTO. - La personalità di Emilio Gabba, storico del mondo antico, viene presentata da numerosi punti di vista: il rigore scientifico, la profonda umanità e la grande disponibilità nei confronti dei giovani, degli studenti in particolare.

$* * *$

ABSTRACT. - The personality of Emilio Gabba, ancient history's scholar, is remembered from various angles: the scientific rigour, the deep humanity and the open-mindedness towards the young people, especially the students.

Sono molto grato all'Istituto Lombardo Accademia di Scienze e Lettere per aver organizzato questa giornata in ricordo di Emilio Gabba, studioso e storico dell'antichità di grande valore, ma soprattutto uomo e maestro di straordinaria personalità.

Desidero quindi ringraziare il Presidente dell'Istituto Lombardo Silvio Beretta, gli organizzatori e i relatori Jean Louis Ferrary, Dario Mantovani, Giancarlo Mazzoli, Francesco Bono, Giuseppe Cambiano. Molti di loro sono docenti all'Università di Pavia e sono stati allievi o colleghi di Emilio Gabba, condividendo con lui riflessioni scientifiche, storiche e culturali.

Come Rettore dell'Ateneo pavese, a me spetta oggi il compito di tracciare il legame tra Emilio Gabba e l'Università di Pavia. Questo iniziò nel 1974 con l'insegnamento di Storia Romana (fino al 1996 quando Gabba fu nominato emerito) e fu alimentato da una lunga, costante e fervida progettazione di iniziative culturali, editoriali, didattiche.

(*) Istituto Lombardo Accademia di Scienze e Lettere, Milano. Rettore dell’Università degli Studi di Pavia, Italia. E-mail: rettore@unipv.it 
Il bel titolo di questo incontro, Humanus, doctus, dilectus, mi guiderà nello svolgere alcune considerazioni su Emilio Gabba e l'Università di Pavia.

Humanus. L'umanità del professor Gabba si è espressa prima di tutto nel suo essere un Maestro. Uomo autorevole, dallo sguardo serio e fermissimo, Emilio Gabba ha saputo trasmettere ai suoi allievi i valori di equità, gentilezza, nobiltà d'animo. Nel favorire il dialogo ha sempre testimoniato la convinta difesa della libertà di pensiero, il rispetto delle buone idee, il rigore (scientifico e morale).

La sua umanità è certamente frutto di una consuetudine radicata nello studio delle bumanae litterae, oltre che di una naturale predisposizione d'animo. L'amore per il mondo antico e i suoi valori è nato in Emilio Gabba a Pavia, al liceo Foscolo ed è poi continuato soprattutto in Università, dove fu allievo di Plinio Fraccaro, con il quale si laureò, a 21 anni, nel giugno del 1948.

Cosa significava per un giovane brillante essere allievo di Plinio Fraccaro?

Significava partire dalla filologia antica e ampliare lo sguardo sul pensiero politico e sui valori civili, per comprendere appieno un'epoca. Un metodo ricordato dallo stesso Gabba in occasione di una lezione al Cedant tenuta nel 2007 al Collegio Borromeo: "il Fraccaro - disse - studiò i frammenti degli oratori come massima espressione dell'attività politica e attraverso i frammenti dell'oratoria, s'intravvedeva chiaro il passaggio alla politica come amministrazione dello stato e come struttura della società". "A tutti gli allievi negli anni quaranta - testimoniava ancora Gabba - era stata suggerita la capacità di ottenere una visione della storia romana attraverso singole figure, che permettevano poi una valorizzazione globale".

Umanità, quindi, come attenzione ai valori dell'uomo: un insegnamento che porterà Emilio Gabba a ricercare costantemente nell'esperienza contemporanea il riflesso della valutazione di problemi antichi.

L'attenzione, il rispetto e l'apprezzamento nei confronti dell'uomo e delle sue idee Emilio Gabba lo ha espresso anche coltivando profondi e radicati rapporti con colleghi, allievi e amici.

Penso ad Arnaldo Momigliano, a Gianfranco Tibiletti (successore di Fraccaro a Pavia), ad Aurelio Bernardi (rettore del Collegio Ghislieri, allievo egli pure di Fraccaro), a Elias Bickerman, Enrica Malcovati, Domenico Magnino, ai molti allievi e colleghi, di tutto il 
mondo, alcuni dei quali presenti qui oggi, a cominciare da Giancarlo Mazzoli e Dario Mantovani.

Proprio quest'ultimo, in un bel ricordo pubblicato su Athenaeum, la prestigiosa rivista nata a Pavia e a lungo diretta da Gabba, sottolinea il profondo significato dell'umanità del professore: "l'impegno di studioso è stato sempre accompagnato da una singolare capacità di docente, esigente e capace al tempo stesso di suscitare interesse e impegno: la sua forma tipica di seminario era la lettura e il commento dei testi, con cui trasmetteva la necessità di mettere il documento al centro di ogni ricerca, e al tempo stesso educava alla libertà dell'interpretazione personale."

Come vedete, il passaggio da bumanus a doctus è immediato: studio e visione della vita sono intimamente legati.

Il professor Gabba non ha insegnato sempre e soltanto a Pavia. Dal 1958 ha insegnato storia antica a Pisa, avviando, con Arnaldo Momigliano, il rinnovamento degli studi di storia antica in Italia. Rientrato a Pavia nel 1974, succedette a un altro allievo di Fraccaro, Gianfranco Tibiletti, alla cattedra di Storia romana nella Facoltà di Lettere e Filosofia.

A Pavia Emilio Gabba ha reso onore alla prestigiosa scuola di Storia romana divenendo uno dei più autorevoli storici dell'antichità e uno dei maggiori intellettuali della seconda metà del Novecento.

Autore di oltre 800 pubblicazioni, è stato Accademico dei Lincei e membro di molte delle più prestigiose accademie internazionali. $\mathrm{Ha}$ diretto Athenaeum e la Rivista Storica Italiana. Ma, soprattutto come professore (chiamato anche a insegnare a Berkeley, a Princeton, a Mainz, a Oxford), ha trasmesso a generazioni di studenti e allievi il senso della storia antica e il significato di valori come la libertà e il dovere, testimoniando un forte attaccamento all'Università e al suo ruolo nella società.

Il contributo di Emilio Gabba alla vita accademica e civile non si esaurisce però qui. L’homo doctus ha per moltissimi anni presieduto la Féderation Internationale des Sociétés des Etudes Classiques, il Centro per la Storia dell'Università e la Società Pavese di Storia Patria che sotto la sua guida ha visto un'autentica fioritura culminata con la pubblicazione a sua cura della Storia di Pavia.

Tra i più recenti riconoscimenti, vi è stata l'elezione nel 2004 a membro onorario dell'American Academy of Arts and Sciences.

In tutti questi ambiti Emilio Gabba ha testimoniato come, grazie alla storia, fatti personaggi e monumenti, altrimenti destinati all'oblio, possono divenire un'esplicita chiave di lettura del presente. 
Alla saggezza dell'umanità e alla sapienza della dottrina lasciatemi, prima di concludere, aggiungere il sorriso dell'bomo dilectus. Emilio Gabba era un amabile conversatore, che coltivava l'amicizia e il piacere di condividere con gli amici i momenti piacevoli della vita. Ma di questo, sono certo, parleranno altri qui presenti.

A me piace ricordarlo negli ultimi anni della sua vita, quando mi capitava di incontrarlo sotto i portici dell'Università. Appoggiato con levità al bastone, si dirigeva ad Athaeneum, o al Cedant, instancabile studioso spesso accompagnato da un allievo o da un collega. Sembrava dire che non c'è traguardo culturale e umano, non c'è ostacolo al vivere quotidiano che non possa essere affrontato con coraggio e determinazione.

Io desidero ringraziarlo per il contributo fondamentale che ha dato alla cultura in generale e all'Università di Pavia in particolare, insegnando a comprendere l'età contemporanea attraverso la ricostruzione dei fenomeni storici antichi. 